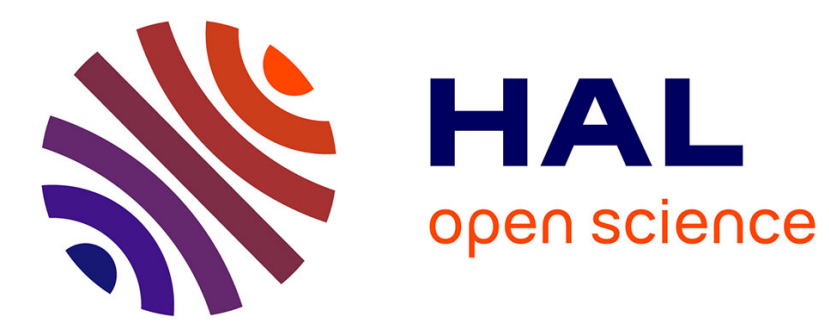

\title{
Ductile Damage and Constraint in Components with Embedded and Surface Semi-Circular Cracks
}

\author{
Zhen Zhang, B. Skallerud
}

\section{To cite this version:}

Zhen Zhang, B. Skallerud. Ductile Damage and Constraint in Components with Embedded and Surface Semi-Circular Cracks. Journal de Physique IV Proceedings, 1996, 06 (C6), pp.C6-173-C6-182. 10.1051/jp4:1996617 . jpa-00254445

\section{HAL Id: jpa-00254445 https://hal.science/jpa-00254445}

Submitted on 1 Jan 1996

HAL is a multi-disciplinary open access archive for the deposit and dissemination of scientific research documents, whether they are published or not. The documents may come from teaching and research institutions in France or abroad, or from public or private research centers.
L'archive ouverte pluridisciplinaire HAL, est destinée au dépôt et à la diffusion de documents scientifiques de niveau recherche, publiés ou non, émanant des établissements d'enseignement et de recherche français ou étrangers, des laboratoires publics ou privés. 


\title{
Ductile Damage and Constraint in Components with Embedded and Surface Semi-Circular Cracks
}

\author{
Z.L. Zhang and B. Skallerud* \\ SINTEF Materials Technology, 7034 Trondheim, Norway \\ * NTNU, Division Applied Mechanics, 7034 Trondheim, Norway
}

\begin{abstract}
Semi-elliptical surface cracks are among the most common defects in welded components subject to fatigue loading. Embedded cracks are also an important type of defects to be considered in welded structures. Control of the static capacity of structures with such defects is important in order to determine load levels corresponding to crack growth initiation and tearing. In the present investigation, the Gurson model based approach has been applied in studying the ductile crack initiation and constraint effect in tension components with semi-circular surface cracks and embedded circular cracks. 3D models of 3 different crack sizes but with exactly the same crack tip element arrangement have been analyzed using the micromechanical parameters established in an earlier study. The results show that for a relatively large embedded crack, the initiation of crack growth will start at the point with the smallest ligament, however, the largest amount of crack growth will occur at the point with the largest ligament. The initiation and growth of surface cracks are rather consistent, with a distinct "canoeing" effect. The influence of crack size and growth on the ductile fracture behaviour is explained by means of constraint.
\end{abstract}

\section{INTRODUCTION}

Recently a 3D numerical study on the combined ductile tearing and fatigue crack growth in an axial loaded flat plate with embedded circular cracks under nominal cyclic plasticity has been performed by the authors [1]. The results showed that when the crack size is small, the fatigue mechanism is dominating the crack growth. However, ductile tearing becomes significant when the crack size has reached a certain level.

In this study, the crack initiation and growth behaviour of circular embedded and semi-circular surface cracks is investigated. The modified Gurson model with the same micro-mechanical parameters used in the previous study [1] has been employed. 3D models with three different crack sizes under remote tension have been analyzed.

It is shown that for the same global deformation, surface cracks initiate earlier and grow faster than the corresponding embedded cracks. For small and medium size embedded cracks, the crack initiation occurs almost simultaneously along the crack front. Nevertheless, the largest amount of crack growth appears in the largest ligament point. For large embedded crack, initiation appears first at the smallest ligament point, because of high plasticity. However, the crack grows faster at the largest ligament point in the early part of crack growth, because of increased constraint. After a certain amount of crack growth, the crack growth at the smallest ligament point will catch up and the crack growth along the crack front becomes almost constant.

The initiation and growth behaviour of surface cracks are rather consistent. For both large and small size cracks, the largest amount of crack growth always occurs at the location $18-40^{\circ}$ away from the surface and the least amount occurs at the surface. The above behaviour is explained by means of plasticity and constraint. 


\section{NUMERICAL PROCEDURES}

\subsection{The modified Gurson model}

Micro-mechanical model based approaches have developed substantially in recent years for predicting the ductile crack growth by void growth and coalescence. In this study the model originally proposed by Gurson [2] and modified by Tvergaard [3,4] and Tvergaard and Needleman [5] was applied. The central part of the Gurson model is the yield function

$\phi(\sigma, f, \bar{\sigma})=\frac{q^{2}}{\bar{\sigma}^{2}}+2 q_{1} f \cosh \left(\frac{3 q_{2} \sigma_{m}}{2 \bar{\sigma}}\right)-1-\left(q_{1} f\right)^{2}=0$

where $q$ is the macroscopic Mises stress formed using the Cauchy stress, $\sigma_{m}$ is the macroscopic mean stress, $\bar{\sigma}$ is the current flow stress, $q_{1}$ and $q_{2}$ are the free parameters introduced by Tvergaard $[3,4], f$ is the current void volume fraction.

There are two parts which contribute to the increase of void volume fraction, the first part is the growth of the existing void, the second part arises from the nucleation of new voids by a straincontrolled or stress-controlled mechanism. In this paper, to be consistent with the previous study [1], a strain controlled void nucleation mechanism is used. The increase of void volume fraction is written:

$d f=(1-f) d \varepsilon_{k k}^{p}+A d \bar{\varepsilon}^{p}$

where $\varepsilon_{k k}^{p}$ represents the sum of normal plastic strain components, $\bar{\varepsilon}^{p}$ is the equivalent plastic strain, $\boldsymbol{A}$ is the void nucleation intensity. The normal distribution model proposed by Chu and Needleman [6] has been used

$$
A=\frac{f_{N}}{S_{N} \sqrt{2 \pi}} \exp \left[-\frac{1}{2}\left(\frac{\bar{\varepsilon}^{p}-\varepsilon_{N}}{S_{N}}\right)^{2}\right]
$$

where $f_{N}$ is the volume fraction of void nucleating particles, $\varepsilon_{N}$ the mean void nucleation burst strain and $S_{N}$ the corresponding standard deviation.

The Gurson model itself cannot properly predict void coalescence. An extra void coalescence criterion has to be implemented. Once the void coalescence starts to occur, the following equation proposed by Tvergaard and Needleman [5] has been used to simulate the post-void coalescence behaviour,

$f^{*}=\left\{\begin{array}{cc}f & \text { for } f \leq f_{c} \\ f_{c}+\frac{f_{u}^{*}-f_{c}}{f_{F}-f_{c}}\left(f-f_{c}\right) & \text { for } f>f_{c}\end{array}\right.$.

Here, $f_{c}$ is the so-called critical void volume fraction at which voids coalesce, $f_{F}$ is the void volume fraction at final failure of the material, and $f_{u}^{*}=1 / q_{1}$.

The Gurson model can predict the ductile crack initiation and growth as long as the associated micro-mechanical parameters are given. Recently it has been shown by Zhang [7] that there is a nonuniqueness problem in the determination of the Gurson parameters. Regarding this, the method for establishing the micro-mechanical parameters by using a physical void coalescence mechanism [8] proposed by Zhang and Hauge [9] has been used in this study. The material used in the paper has a yield 
stress of $370 \mathrm{MPa}, E=210000 \mathrm{MPa}, v=0.3$. The true stress-strain curve is reported in [1] and can be approximately fitted by a plastic hardening exponent $n=0.12$. The Gurson parameters are: $f_{0}=0.00033$, $f_{N}=0.006, f_{c}=0.026, f_{F}=0.15, \varepsilon_{N}=0.3, S_{N}=0.1, q_{1}=1.5, q_{2}=1.0$. These parameters are established by fitting the numerical result with the experimental load-displacement curve of a tensile specimen. Details of the parameter fitting can be obtained from [1].

\subsection{FE models}

Both semi-circular surface and circular embedded cracks with three different crack sizes, $a / w=0.3,0.5$ and 0.7 , where $a$ is the crack size, $w$ is the thickness of the plate, have been analyzed. For surface and embedded cracks with the same $a / w$ ratio, the same mesh but different boundary conditions have been used. Fig. 1a shows the mesh for the $a / w=0.5$ cracks in a global scale and Fig. $1 \mathrm{~b}$ shows the basic $2 \mathrm{D}$ mesh used for creating the 3D meshes along the crack front. Regular elements are used in the crack plane. The mesh size $(D)$ in the vicinity of crack tip is $0.033 \mathrm{~mm}$. The mesh arrangement at the crack front for different crack models is exactly the same in order to avoid the extra problem introduced by mesh size. The dimensions of the FE model of the plate are $10 \times 30 \times 60 \mathrm{~mm}$, which is $1 / 8$ of the plate with embedded cracks and $1 / 4$ of the plate with surface cracks. 20-node brick elements with reduced integration and large deformation formulation as implemented in ABAQUS were used in the analyses. There are about 800 elements and 4000 nodes for each crack model. It takes about 3 CPU hours for each analysis on a CRAY J90 computer.

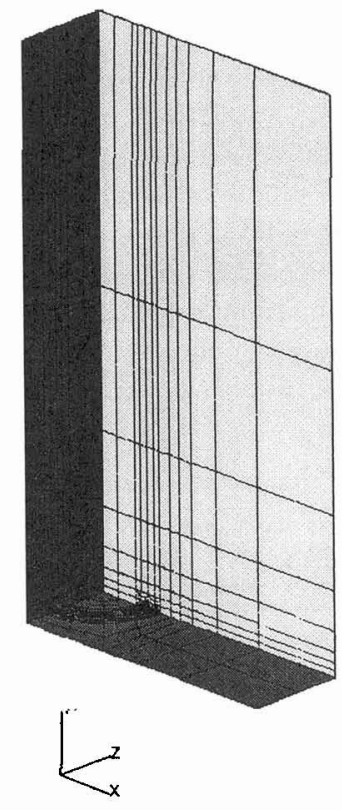

(a)

(a)
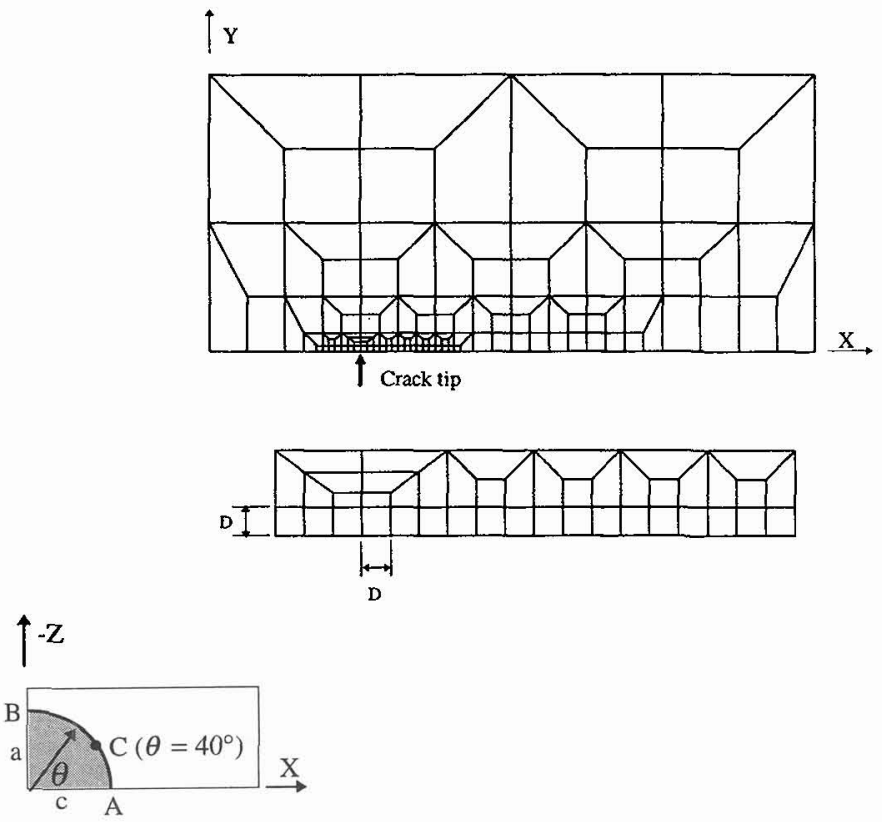

(b)

Fig. 1 a) $3 D$ mesh for the $a / w=0.5$ cracks, b) basic $2 D$ mesh used for creating the $3 D$ meshes. 


\subsection{Numerical implementation of the Gurson model and numerical crack growth}

The Gurson model introduced in section 2.1 was implemented into ABAQUS via UMAT subroutine using the numerical procedure developed by Zhang and Niemi [10]. In this paper, a numerical crack is defined as the part of material where the load carrying capacity has disappeared, i.e. $f=f_{F}$. There are different ways of defining numerical cracks [11]. Different definitions can affect the crack initiation time but may not significantly affect the crack growth behaviour as long as a consistent definition is used.

\section{RESULTS}

Because a large deformation formulation is used, the $J$-integral becomes path dependent after some amount of crack growth. Therefore, the $J$ is not used as a loading parameter. Only the $J$ values at crack initiation were compared. In all the results, the crack growth and radial distance to the crack tip are normalized by the element size $(D)$.

\subsection{Crack size effect on damage development}

The void volume fractions as a function of the global strain at the most critical integration point of the first element ahead of crack tip at point A (largest ligament) are shown in Fig. 2a for the three embedded cracks. It should be noted that for the $3 \mathrm{~mm}$ crack, there is no crack growth even when the nominal strain is larger than $3.4 \%$. However, according to the damage model, the ductile tearing starts to appear for the $7 \mathrm{~mm}$ crack model when the global strain has reached about $0.5 \%$. The results in Fig. 2a can support the findings in [1]. The actual maximum global strain in the test $[1,12]$ was $1.3 \%$. Fig. 2a shows that when the crack size is small, ductile tearing by void growth and coalescence is not possible for the $a / w=0.3$ crack. Ductile tearing becomes possible within the applied nominal strain range only when the crack size is larger than $5 \mathrm{~mm}$.

The void volume fractions at point $\mathrm{C}\left(\theta=40^{\circ}\right)$ for the three surface cracks are shown in Fig. $2 \mathrm{~b}$. The crack size effect on the damage development in the surface cracks has the same trend as that in the embedded cracks. Comparing Fig. $2 a$ and $2 b$, it can be seen that for the same global strain, damage develops faster in the surface cracks than the corresponding embedded cracks. So does the crack growth.

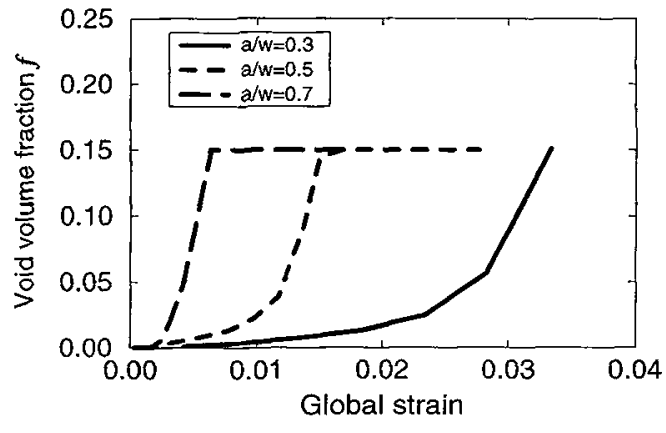

(a)

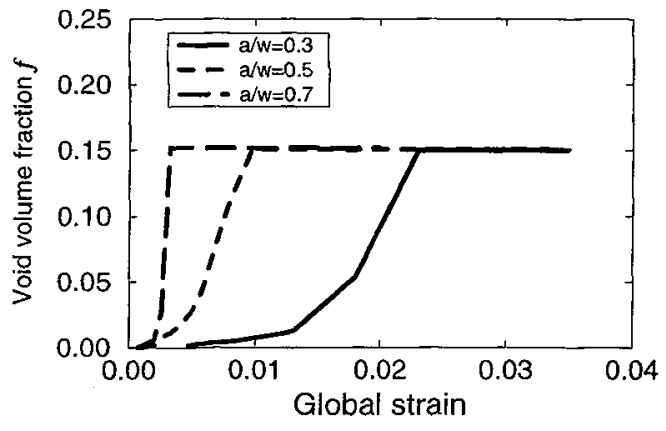

(b)

Fig. 2 a) Damage development versus global strain at the most critical integration point at location $A$ for the embedded cracks; b) at location $\mathrm{C}$ for the surface cracks. 


\subsection{Crack initiation}

Crack initiation $\left(f=f_{c}\right)$ is examined for both surface and embedded cracks. The distribution of averaged void volume fraction in the first elements along the crack front at crack initiation is shown in Fig. 3a for the embedded cracks. It can be seen that for the small and medium embedded cracks, the crack initiation occurs almost simultaneously along the crack front. For the large embedded crack, the void volume fraction at point B (smallest ligament) is significantly higher than that at point A (largest ligament). Therefore, the crack initiation occurs first at point $B$. This is due to the fact that the small ligament in point $B$ results in high plasticity which accelerated void growth.

The distribution of the averaged void volume fraction for the surface cracks is shown in Fig. $3 \mathrm{~b}$. Compared with the embedded cracks, the distribution of void volume fraction for the surface cracks is zigzag. This is probably due to the much stronger stress-strain gradient along the crack front in the surface cracks than that in the embedded cracks. Another reason is that in the analyses only four elements along the crack front (A-B) were used. The general observation is that the maximum damage does not occur at the largest ligament point (A) neither in the smallest ligament point (B), but at the location with an angle $18-40^{\circ}$ away from the surface.

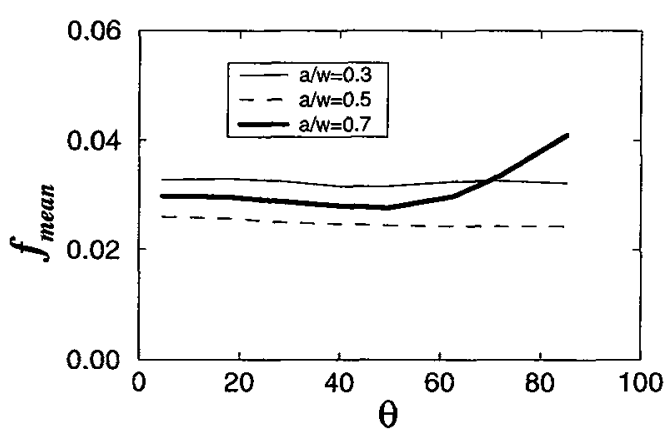

(a)

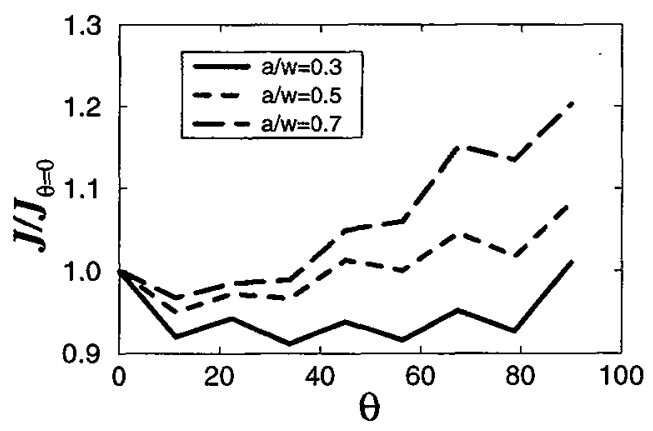

(c)

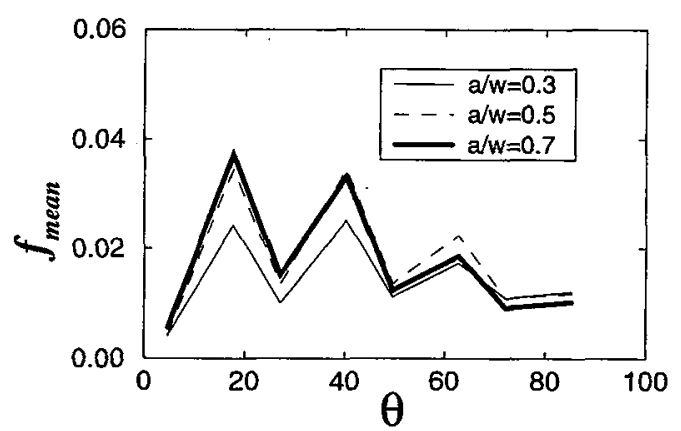

(b)

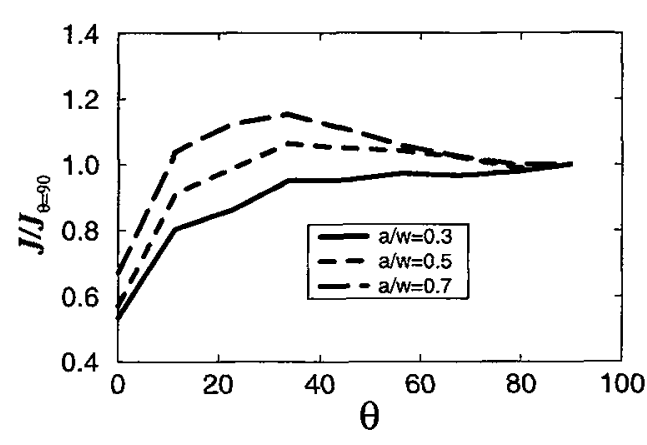

(d)

Fig. 3 a) The distribution of the averaged void volume fraction in the crack front elements at crack initiation for the embedded cracks, b) for the surface cracks, c) $J$ distribution along the crack front at crack initiation (same global deformation levels as in Fig. 3a) for the embedded cracks, d) $J$ distribution for the surface cracks (same global deformation levels as in Fig. 3b). 
The normalized $J$ integral at crack initiation for the embedded and surface cracks are shown in Fig. 3c and $3 \mathrm{~d}$, respectively. A similar observation as the void volume fraction can be seen. For the large embedded crack, largest $J$ appears at point B. The locations of the largest $J$ in the medium and large surface cracks are consistent with the locations of the largest damage $(f)$. However, for the small surface crack, largest $J$ occurs at the smallest ligament point (centre).

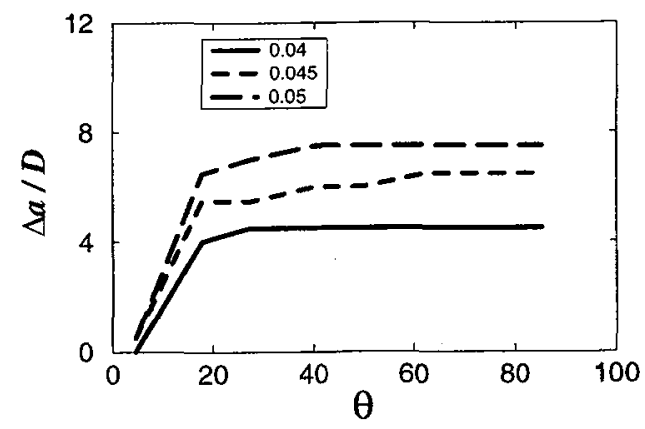

(c)

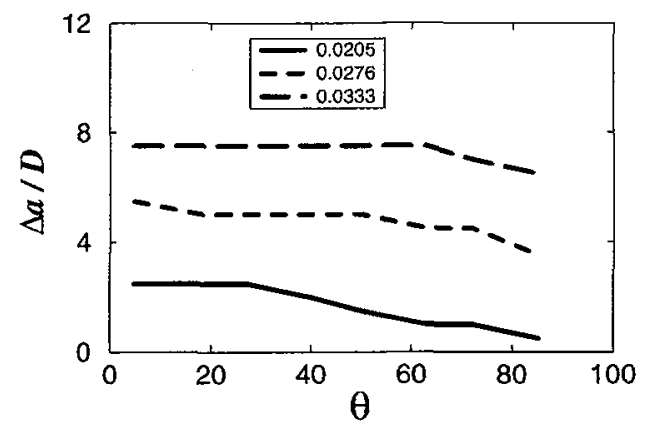

(a)

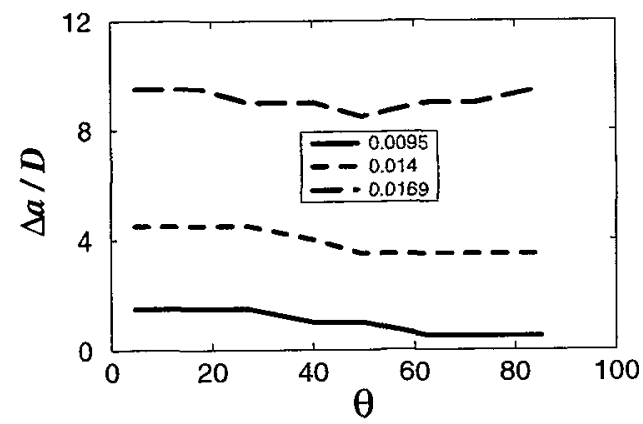

(b)

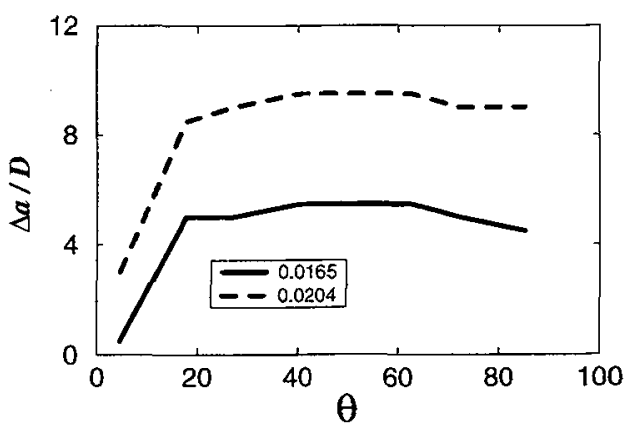

(d)

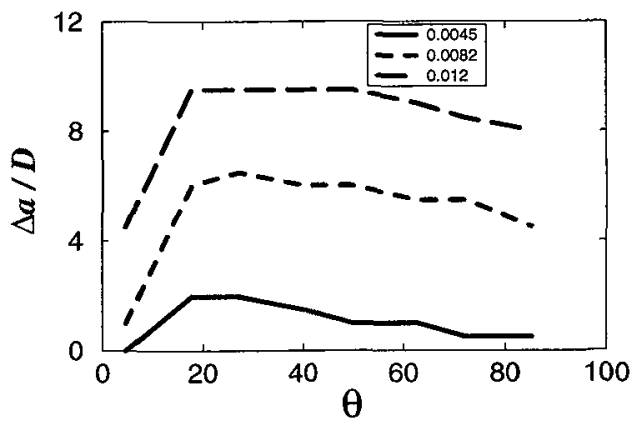

(e)

Fig. 4 Crack growth distribution for a) $a / w=0.5$ embedded crack, b) $a / w=0.7$ embedded crack, c) $a / w=0.3$ surface crack, d) $a / w=0.5$ surface crack and e) $a / w=0.7$ surface crack. The data in the legend indicate the global strain level. 


\subsection{Crack growth - embedded cracks}

The crack growth along the crack front for the embedded cracks $a / w=0.5$ and 0.7 at different global deformation levels is shown in Fig. $4 \mathrm{a}$ and $4 \mathrm{~b}$. For the $a / w=0.3 \mathrm{crack}$, no crack growth was observed within the applied global strain up to $3.4 \%$. For the $a / w=0.5 \mathrm{crack}$, the crack grows faster at point $\mathrm{A}$ in the early part of crack growth. However, point B gradually catches up with the same amount of crack growth as point $\mathrm{A}$. It is interesting to note that for the $a / w=0.7$ crack, the crack grows faster also at point A, although the largest amount of damage occurred at point B at crack initiation (Fig. 3a). After some amount of crack growth $(\approx 4 D)$, the crack growth at point $B$ will catch up with the amount of crack growth at point $\mathrm{A}$. This behaviour can be explained by the results shown in Fig. 5 .

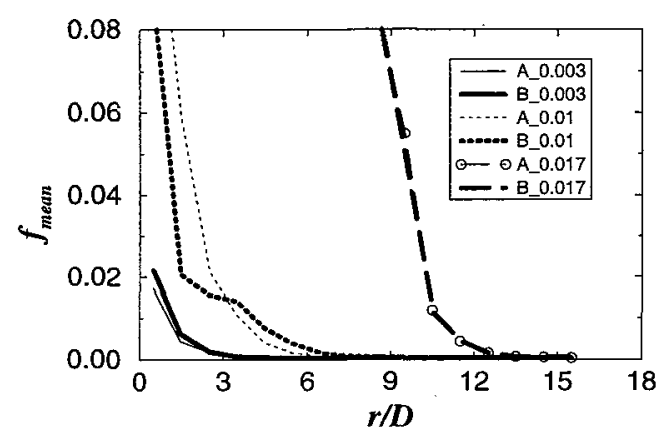

(a)

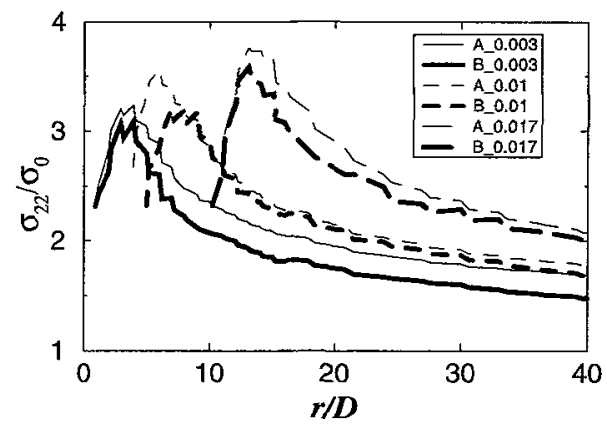

(b)

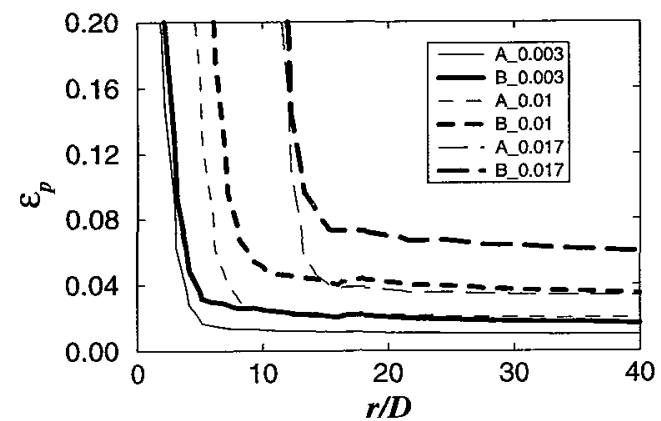

(c)

Fig. 5 a) The distribution of the averaged void volume fraction, b) normalized opening stress, and c) equivalent plastic strain at point $\mathrm{A}$ and $\mathrm{B}$ for the $a / w=0.7$ embedded crack.

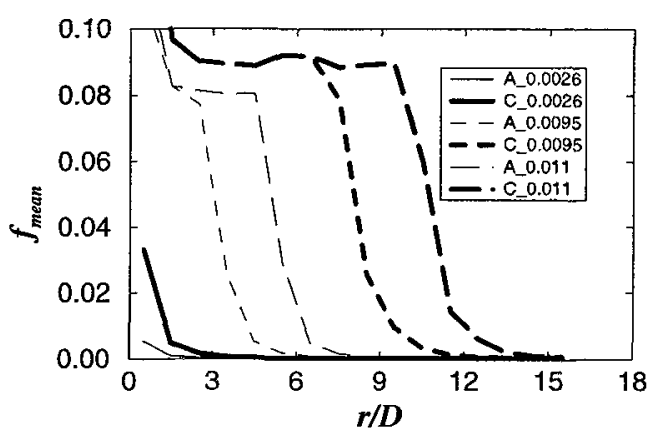

(a)

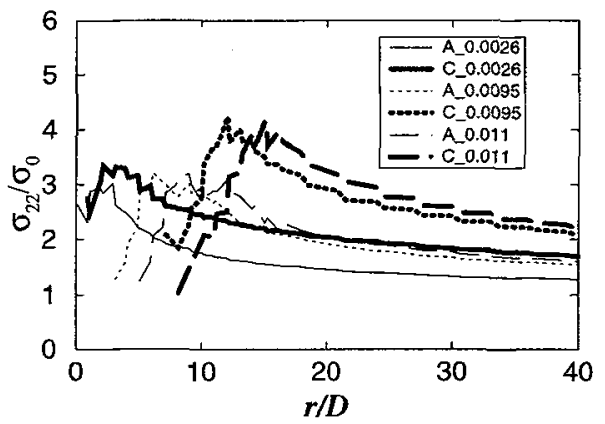

(b)

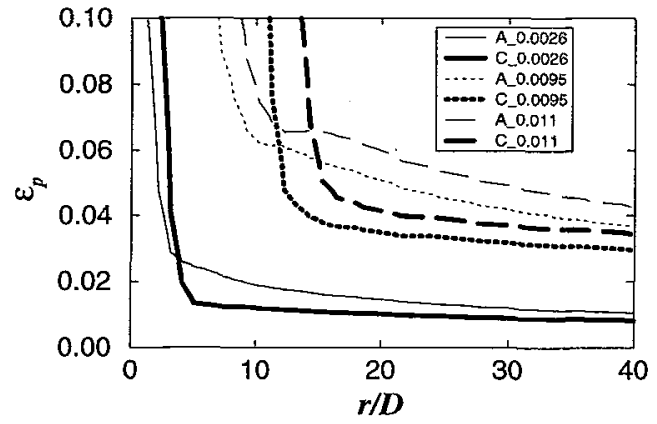

(c)

Fig. 6 a) The distribution of the averaged void volume fraction, b) normalized opening stress, and c) equivalent plastic strain at point $A$ and $C$ for the $a / w=0.7$ surface crack. 
Fig. 5 shows the comparison of void volume fraction, normalized opening stress and equivalent plastic strain ahead of the crack tip at different global deformation levels for the $a / w=0.7$ crack. Fig. $5 c$ shows that before the crack growth (global strain 0.003), the plastic strain at point $B$ is higher than that at point $A$, because of smaller ligament. The opening stress level at point $A$ is slightly higher than that at point $B$. It can be seen from Fig. 5a that the damage at point $B$ is higher than that at point $A$, which implies that the plastic deformation is dominating the damage development. However, when the crack growth has started, the opening stress at point A continues to increase (constraint increase) and the stress level at point B is almost constant (see Fig. 5b at the global strain level 0.01). This increased opening peak stress at point $A$ played a significant role in void growth and accelerated the damage development. Therefore, we observe larger amount of crack growth (Fig. $4 \mathrm{~b}$ ) at point A. It is interesting to note that this trend of crack growth is dependent on the total amount of crack growth. Fig. 5b shows that the peak stress at point B is also elevated but after some amount of crack growth, although still smaller than that at point $\mathrm{A}$. Combing the larger plastic deformation and the later elevated opening stress, the damage at point $B$ is accelerated and the amount of crack growth at point $B$ finally catches up with that at the point A (Fig. $4 \mathrm{~b}$ and $5 \mathrm{a}$ ).

The general observation in the embedded cracks is that when the total amount of crack growth is small $(\leq 4 D)$, the largest amount of crack growth occurs at point $A$. The amount of crack growth at point $B$ catches up with that at point A when the total amount of crack growth increases. Fig. 7 schematically shows the crack growth behaviour of the embedded cracks. This observation could explain the crack shape development reported in [12]. In [12], it has been clearly seen that the radius of the final embedded crack at point $\mathrm{A}$ is larger than that at point $\mathrm{B}(c / a \approx 1.1)$. Recent analyses [1] of the results in [12] show that the fatigue mechanism is dominating the crack growth when the crack is small. Both metallographic examination of the fracture surface and numerical investigation indicate that ductile tearing by void growth and coalescence becomes a significant contribution to the total crack growth when the crack radius has reached about $5 \mathrm{~mm}$. The maximum nominal strain applied in the test is $1.3 \%$. From Fig. 4 , we can see that for the $a / w=0.7$ crack the total amount of crack growth at the maximum nominal strain is still small $(\leq 4 D)$, therefore, crack grows faster at point $A$ than that at point $\mathrm{B}(c / a>1.0)$.
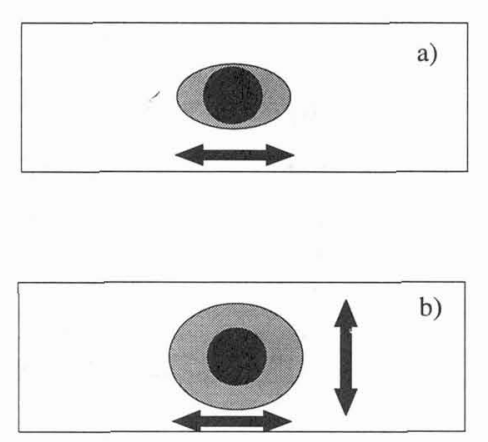

Fig. 7 Schematic plots of the crack growth behaviour of embedded cracks: when the amount of crack growth is small a) and when the amount of crack growth is large b). Black areas represents the original carcks.
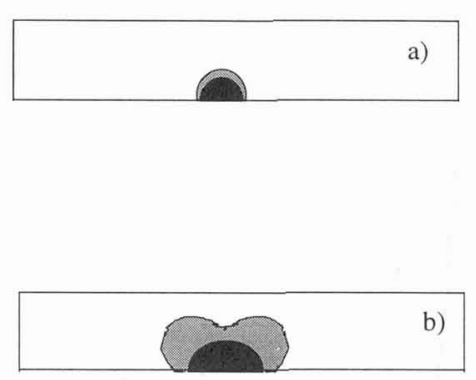

Fig. 8 Schematic plots of the crack growth behaviour of surface cracks: when the crack size is small a) and when the crack size is large b). Black areas represents the original carcks. 


\subsection{Crack growth - surface cracks}

The crack growth at different deformation levels for the surface cracks are shown in Fig. 4c, $4 \mathrm{~d}$ and $4 \mathrm{e}$ for the $a / w=0.3,0.5$ and 0.7 cracks, respectively. It can be seen that the least amount of crack growth occurs at the surface (point A). This observation is independent of the crack size. For the $a / w=0.3 \mathrm{crack}$, the amount of crack growth along the crack front is almost constant except point A. A significant "canoeing" effect $[13,14]$ can be seen for the $a / w=0.7$ crack. The "canoeing" becomes less significant in the $\mathrm{a} / \mathrm{w}=0.5 \mathrm{crack}$. Furthermore, for the $a / w=0.7 \mathrm{crack}$, the "canoeing" is more distinct in the beginning of crack growth. It becomes less distinct with the increase of crack growth.

The void volume fraction, normalized opening stress and equivalent plastic strain at point $\mathrm{A}$ and $\mathrm{C}$ for the $a / w=0.7$ crack at different deformation levels are compared in Fig. 6 . The plastic strain ahead of the peak stress is higher at Point $\mathrm{A}$ than at point $\mathrm{C}$. Behind the peak stress, the plastic strain at point $\mathrm{C}$ increases dramatically because of the damage and crack growth. Fig. $6 \mathrm{~b}$ shows that the opening stress level at point $C$ is higher than that at point $A$. The difference between the opening stress levels at point $\mathrm{C}$ and point $\mathrm{A}$ increased after the ductile crack growth has started. It can be seen that the opening stress (constraint level) at point $\mathrm{C}$ increases significantly after ductile growth. The combined effect of plastic strain and increased opening stress level can be seen in Fig. 6a. At the same distance to the initial crack tip, the damage at point $C$ is always higher than that at point $A$, which indicates that the crack grows always faster at point $\mathrm{C}$ than at point $\mathrm{A}$.

\section{DISCUSSION AND CONCLUDING REMARKS.}

By using 3D FE models and applying the modified Gurson model, the crack initiation and growth behaviour for both embedded and surface cracks have been investigated and compared. It has been shown that crack growth depends on the initial crack size, location and the total amount of crack growth. For small and medium embedded cracks, crack initiation occurs almost simultaneously along the crack front. For a large embedded crack, the crack initiation will occur at the smallest ligament point. This is due to the high plasticity at the smallest ligament point. After the crack growth has started, the constraint at the largest ligament point increases significantly while the constraint level in the smallest ligament is more or less constant. This increased constraint results in larger crack growth at the largest ligament point. However, the constraint level at the smallest ligament will also increase after some amount of crack growth $(\approx 4 D)$ and the crack growth at the smallest ligament point finally catches up with the crack growth at the largest ligament.

The constraint increase after ductile crack growth has recently been reported by others [15]. The direct consequence of the increased constraint is the increased likelihood of cleavage fracture. It should be kept in mind that the constraint increase depends on the material plastic hardening capability and the initial damage parameters. The effect of these two factors has not been explored in the present investigation.

For surface cracks, the "canoeing" behaviour $[13,14]$ has been predicted for both the $a / w=0.5$ and 0.7 cracks, especially when the total amount of crack growth is small. In general, the crack growth behaviour of the surface cracks depends on crack size. Fig. 8 schematically plots the crack growth behaviour of the surface cracks. Although the equivalent plastic strain at the surface (point $\mathrm{A}$ ) is higher than that at point $\mathrm{C}$, the higher stress level at point $\mathrm{C}$ results in larger amount of crack growth.

Damage is a combination of the contribution by both plastic strain and stress triaxility. The results of embedded cracks show that short ligament and high plasticity does not necessarily lead to larger ductile crack growth. In the present investigation, we observe that a high stress level plays a more significant role in void growth than high plastic strain.

In this study, the strain-controlled void nucleation has been used and the mesh size effect was not studied. It should be mentioned that the void nucleation burst strain, $\varepsilon_{N}=0.3$ was applied in the analyses. From Fig. 5c and 6c, we observe that the plastic strain ahead of the peak stress is very small 
compared with the value of the burst strain, hence, the role of void nucleation in the increase of void volume fraction should not be significant before the peak stress has reached. In further studies, cases with stress-controlled void nucleation and cases without void nucleation will be compared.

\section{References}

[1] Skallerud B. and Zhang Z. L., Int. J. Solids Struct. (1996) in press.

[2] Gurson A. L. Plastic flow and fracture behavior of ductile materials incorporating void nucleation, growth, and interaction, Ph.D Dissertation (1975), Brown University.

[3] Tvergaard V., Int. J. Fract. 17 (1981) 389-407.

[4] Tvergaard V., Int. J. Fract. 18 (1982) 237-252.

[5] Tvergaard V. and Needleman A., Acta Metall. 32 (1984) 157-169.

[6] Chu C. C. and Needleman A., J. Engng Mater. 102 (1980) 249-256.

[7] Zhang Z. L., Fatigue Fract. Engng. Mater. Struct. 19 (1996) 561-570.

[8] Zhang Z. L. and Niemi E., Int. J. of Fract. 70 (1995) 321-334.

[9] Zhang Z. L. and Hauge M., "Fitting the Gurson parameters by using a physical void coalescence mechanism", ECF11-Mechanisms and Mechanics of Damage and Failure, 1996.

[10] Zhang Z. L. and Niemi E., Int. J. Numer. Meth. Engng 38 (1995) 2033-2053.

[11] Xia L. and Shih C. F., J. Mech. Phys. Solids 43 (1995) 233-259.

[12] Skallerud B., Fatigue Fract. Engng. Mater. Struct. 15 (1992) 43-56.

[13] Klemm W., Memhard D. and Schmitt W., "Experimental and numerical investigation of surface cracks in plates and pipes", Fracture Mechanics Verification by Large-Scale Testing, EGF/ESIS8, edited by K. Kussmaul, Mechanical Engineering Publications, London, 1991, pp. 139-150.

[14] Brocks W., Kunecke G., Noack H.-D. and Veith H., Nuclear Engng Design 112 (1989) 1-14.

[15] Ruggieri C. and Dodds R., Structural Research Series No. 606, University of Illinois at UrbanaChampaign, 1995. 